\title{
Dynamics of sweeping through an instability: Passage-time statistics for colored noise
}

\author{
M. C. Torrent \\ Departament d'Estructura i Constituents de la Materia, Facultat de Física, Universitat de Barcelona, \\ Diagonal 647 E-08028 Barcelona, Spain \\ F. Sagués \\ Departament de Química Física, Facultat de Química, Universitat de Barcelona, \\ Diagonal 647, E-08028 Barcelona, Spain \\ M. San Miguel \\ Departament de Física, Universitat de les Illes Balears, E-07071 Palma de Mallorca, Spain
}

(Received 28 July 1989)

\begin{abstract}
A calculation of passage-time statistics is reported for the laser switch-on problem, under the influence of colored noise, when the net gain is continuously swept from below to above threshold. Cases of fast and slow sweeping are considered. In the weak-noise limit, asymptotic results are given for small and large correlation times of the noise. The mean first passage time increases with the correlation time of the noise. This effect is more important for fast sweeping than for slow sweeping.
\end{abstract}

\section{INTRODUCTION}

Our interest in this paper is the description of a general situation in which a system undergoes an instability when the appropriate control parameter is continuously swept from below to above the instability point. The basic question addressed in the determination of the time at which the instability is observed. This question appears in two alternative situations. One of them, associated with slow sweeping rates, aims at determining a dynamical bifurcation point. Such a dynamical instability is known to be delayed with respect to the static instability point. Recent reviews on delayed bifurcations are available. ${ }^{1}$ A second situation is associated with fast sweeping rates. It appears in studies of transient dynamics describing the relaxation from unstable states. Both situations have been recently analyzed in some detail in the context of the dynamics of the laser switch-on. ${ }^{2-7}$ Experimental results are available for $\mathrm{Ar}^{+}$lasers, ${ }^{2}$ semiconductor lasers, ${ }^{4,6} \mathrm{CO}_{2}$ lasers, ${ }^{5}$ and $\mathrm{CO}_{2}$ with saturable absorber. ${ }^{7}$ A general point of view $^{3}$ to address the problem of the dynamics when crossing an instability is to identify it as a stability problem which, in a dynamical sense, is associated with the concept of the lifetime of a state. The lifetime is determined by fluctuations and it can be calculated by stochastic methods as a mean first passage time. In this paper we follow this point of view extending our previous analysis ${ }^{3}$ to the case in which the noise-which is responsible for the decay of the state becoming unstable - has a finite correlation time (colored noise). Although our general methodology and results have a broad applicability, we present our analysis, for definiteness, in the context of the dynamics of the laser switch-on.

The stochastic analysis of the problem at hand admits two alternative approaches. In the first approach, the focus is on the calculation of the time-dependent moments of the relevant variables. ${ }^{8,9}$ For instance, in the laser case one calculates the time-dependent intensity. In the second approach, one follows the individual stochastic realizations of the process up to a given prescribed value of the intensity. One can then evaluate the statistics of the time at which the individual realizations reach that value of the intensity. ${ }^{10} \mathrm{~A}$ general comparison of the two approaches in the case of white noise is given in Ref. 11. The second approach has been followed in Refs. 3-6. Its most important advantage is that all the statistical properties of the passage time can be calculated and not only a mean characteristic time scale to reach a prescribed intensity value. ${ }^{9}$ For example, the variance of the passage-time distribution is associated with the time jitter of the laser switch-on ${ }^{4}$ and it also gives an indirect way of measuring the total gain. ${ }^{5}$ However, useful results for passage-time statistics are generally obtained only in the weak-noise limit. Another important question concerning a stochastic analysis of this sort refers to the preparation of the system (initial conditions). We will follow here the criterion ${ }^{3}$ that the system is initially in a steady state determined by the noise acting on the system. An initial condition specified independently of the noise was considered in Ref. 8 and a comparison between the two situations is given in Ref. 9. Such preparation effects are more important in the colored noise situation considered here due to the non-Markovian character of the dynamics. ${ }^{12}$ As a separate matter which we will not discuss further, we mention that the consequences of having an initial state very close to threshold have been analyzed in Ref. 6.

The problem of the sweeping dynamics involves several time scales that have to be considered simultaneously. These are fixed by the noise intensity $\epsilon$, the square root of the sweeping rate $\alpha^{1 / 2}$, the final value of the pump pa- 
rameter $a$, and the inverse of the correlation time of the noise $\tau^{-1}$. We will always consider the weak-noise limit in which $\epsilon^{-1}$ is the largest time scale of the problem. Passage-time statistics have already been calculated for three limiting cases. The first one corresponds to the well-known instantaneous switch-on $(\alpha \rightarrow \infty)$ in the white noise limit $(\tau=0){ }^{10,13}$ The second situation ${ }^{12}$ considers an instantaneous switch-on $(\alpha \rightarrow \infty)$ with colored noise $(\tau \neq 0)$. This case corresponds to $\alpha^{1 / 2} \tau \rightarrow \infty$. Finally, a third case $\mathrm{e}^{3}$ is the one of finite sweeping with white noise $(\tau=0)$. It corresponds to $\alpha^{1 / 2} \tau=0$. In this paper we analyze situations with $\alpha^{1 / 2} \tau$ finite in the cases of fast sweeping $a \lesssim \alpha^{1 / 2}$ and slow sweeping $\alpha^{1 / 2}<<$.

The outline of the paper is the following. In Sec. II we define our model, we set the opportune notation, and we review the instantaneous switch-on case with colored noise for later reference. Section III is devoted to fast sweeping situations. This seems to be the case of interest for switch-on of $\mathrm{CO}_{2}$ lasers in Ref. 5. The variance of the passage-time statistics turns out to be independent of $\tau$ in this limit. The mean passage time increases monotonically with $\tau$ and decreases with $\alpha$. We find that the first correction to the white noise limit is of order $\left(\alpha^{1 / 2} \tau\right)^{2}$. In the limit of large correlation time $\alpha^{1 / 2} \tau \gg 1$ the mean first passage time diverges logarithmically with $\alpha^{1 / 2} \tau$ for $a \tau \gg 1$. Results related to the instantaneous limit are also given. In Sec. IV we discuss the case of slow sweeping $\alpha^{1 / 2}<a$. In this case the variance of the passagetime statistics is reduced with respect to the white noise limit. The qualitative effect of the finite correlation time of the noise is the same as for the fast sweeping situation, both for small and large $\tau$, but its quantitative consequences are now much weaker. In Sec. IV we discuss the slow sweeping case. Our results for the mean first passage time are related to an analysis for the time dependence of the mean intensity. ${ }^{9}$ Actually, a close comparison does not seem feasible, not only because of the different definition of the characteristic time, but also due to the different parametrization of the problem. However, a basic agreement exists in the general importance of preparation effects and the scaling with $\alpha^{1 / 2}$ as well as in the results concerning the growth of the characteristic time with $\alpha^{1 / 2} \tau$ for $\alpha^{1 / 2} \tau \ll 1$ and its divergence for $\alpha^{1 / 2} \tau \gg 1$. Two appendixes contain details of the mathematical calculations.

\section{LASER THRESHOLD MODEL AND INSTANTANEOUS SWITCH-ON}

Our analysis is based on the following model equation for the evolution of the complex electric field $E=E_{1}+i E_{2}$ corresponding to a single model laser in the good cavity limit:

$$
\partial_{t} E=a(t) E+N(E)+\epsilon^{1 / 2} \xi(t) .
$$

The parameter $a(t)$ is the net gain parameter and $N(E)$ represents the nonlinear saturation terms. The parameter $\epsilon$ measures the strength of the complex noise $\xi(t)=\xi_{1}(t)+i \xi_{2}(t)$. In the good cavity limit we consider a reduced dynamics for the field, obtained after an adiabatic elimination of other variables. The noise driving the instability $\xi(t)$ is assumed to be Gaussian with zero mean and correlations

$$
\left\langle\xi_{i}(t) \xi_{j}\left(t^{\prime}\right)\right\rangle=\delta_{i j} \frac{1}{2 \tau} e^{-\left|t-t^{\prime}\right| / \tau}, \quad i, j=1,2
$$

where $\tau$ is the correlation time of the noise. The origin of a nonvanishing correlation time may be diverse. Before taking a good cavity limit, the Langevin forces appearing in the field equation are associated with external noise sources and thermal background radiation. ${ }^{14,15}$ On the other hand, spontaneous-emission noise is modeled by white noise added to the polarization equation. ${ }^{14,15}$ Nonwhite noise in (2.1) can then be associated either to external noise or to spontaneous emission since the elimination of the polarization variable introduces, in principle, a nonwhite noise in the reduced dynamics for the electric field.

We will consider a dynamical instability in which $a(t)$ varies from below to above threshold

$$
a(t)=\left\{\begin{array}{c}
a_{0}<0, \quad t<0 \\
a_{0}+\alpha t, \quad 0<t<t_{1} \\
a, \quad t>t_{1}
\end{array}\right.
$$

In a static sense, the instability would occur at the time $\bar{t}=\left|a_{0}\right| / \alpha$ such that $a(\bar{t})=0$. The dynamical instability has been considered in a deterministic framework and also in the white noise limit $\tau=0$ in Ref. 3. We are here mainly concerned with the role of the parameter $\tau$, in the onset of this dynamical instability. We are interested in describing the switch-on process. The time at which the intensity takes an observable value is identified with the lifetime of the unstable state. This is known to be largely independent of the nonlinear terms in (2.1), which are essential to describe the saturation to the final state. Our analysis is, thus, independent of the nonlinearities.

The linearized version of (2.1) admits the solution

$$
E_{i}(t)=h_{i}(t) \exp \left(\int_{0}^{t} a\left(t^{\prime}\right) d t^{\prime}\right), \quad i=1,2
$$

where $h_{i}(t)$ plays the role of an effective random initial condition

$$
h_{i}(t)=E_{i}(0)+\epsilon^{1 / 2} \int_{0}^{t} \xi_{i}\left(t^{\prime}\right) \exp \left(-\int_{0}^{t^{\prime}} a\left(t^{\prime \prime}\right) d t^{\prime \prime}\right) d t^{\prime}
$$

The initial value of the field $E_{i}(0)$ is itself a random variable. The system is assumed to be at $t=0$ in a stationary state associated with the value $a_{0}<0$ of the pump parameter. For times $t<0, E_{i}(t)$ satisfies the equation

$$
\partial_{t} E_{i}(t)=a_{0} E_{i}(t)+\epsilon^{1 / 2} \xi_{i}(t),
$$

so that 


$$
E_{i}(0)=\lim _{s \rightarrow-\infty} \epsilon^{1 / 2} \exp \left[\int_{s}^{0} a_{0} d s^{\prime}\right) \int_{s}^{0} \xi_{i}\left(s^{\prime}\right) \exp \left(-\int_{s}^{s^{\prime}} a_{0} d s^{\prime \prime}\right) d s^{\prime} .
$$

The complex number $h=h_{1}+i h_{2}$ is described by a bivariate Gaussian process. The stochastic properties of the intensity $I(t)=|E(t)|^{2}$ are determined by the stochastic properties of $h^{2}=h_{1}^{2}+h_{2}^{2}$ :

$$
I(t)=h^{2}(t) \exp \left[2 \int_{0}^{t} a\left(t^{\prime}\right) d t^{\prime}\right] \text {. }
$$

The strategy we follow to calculate the passage-time statistics corresponding to a prescribed intensity value $I$ consists in inverting (2.8), obtaining $t$ as a random function of $I$. The procedure is then formally the same as in the white noise limit $\tau=0$. The difference arises as a consequence of the different form adopted by $h^{2}(t)$.

The bivariate Gaussian distribution $P\left(h_{1}, h_{2}\right)$ is completely determined by $\left\langle h^{2}(t)\right\rangle$. This quantity evolves dynamically according to a differential equation

$$
\partial_{t}\left\langle h^{2}(t)\right\rangle=2 \epsilon \exp \left[-\int_{0}^{t^{\prime}} a\left(t^{\prime \prime}\right) d t^{\prime \prime}\right]\left[e^{-t / \tau} \frac{1}{1+\left|a_{0}\right| \tau}+\frac{1}{\tau} \int_{0}^{t} e^{-\left(t-t^{\prime}\right) / \tau} \exp \left[-\int_{0}^{t^{\prime}} a\left(t^{\prime \prime}\right) d t^{\prime \prime}\right] d t^{\prime}\right],
$$

whose integration enables us to calculate $\left\langle h^{2}(t)\right\rangle$ at any time. The origin of the first term in the right-hand side (rhs) of (2.9) should be remarked. It appears due to the correlation of the initial condition $E_{i}(0)$ with the noise acting for $t>0$ :

$$
\left\langle E_{i}(0) \xi_{i}(t)\right\rangle=\frac{\epsilon^{1 / 2}}{2\left(1+\left|a_{0}\right| \tau\right)} e^{-t / \tau}
$$

This correlation is a genuine non-Markovian effect of the colored noise considered here. Equation (2.9) has to be solved with initial conditions determined according to (2.7),

$$
\left\langle h^{2}(0)\right\rangle=\langle I(0)\rangle=\frac{\epsilon}{\left|a_{0}\right|\left(1+\left|a_{0}\right| \tau\right)} .
$$

Our general scheme, developed in the following sections, consists in the explicit solution of $(2.9)-(2.11)$ for different situations. We find that for times of interest $\left\langle h^{2}(t)\right\rangle$ can be approximated by $\left\langle h^{2}(\infty)\right\rangle$, so that inverting (2.8) the generating function $W(\lambda)=\left\langle e^{-\lambda t}\right\rangle$ for the passage-time statistics can be calculated. The moments $\left\langle t^{n}\right\rangle$ of the passage-time distribution are calculated by simply differentiating $W(\lambda)$ with respect to $\lambda$ at $\lambda=0$. The results in terms of $\left\langle h^{2}(\infty)\right\rangle$ are formally the same as for $\tau=0$. The effects of having $\tau \neq 0$ are actually contained in the explicit result for $\left\langle h^{2}(\infty)\right\rangle$.

A limiting case which is of interest for later reference and comparison corresponds to an instantaneous switch $\left(\alpha \rightarrow \infty, t_{1} \rightarrow 0^{+}\right) .{ }^{12}$ In such a case we have

$$
\left\langle h^{2}(t)\right\rangle=\left\langle h^{2}(0)\right\rangle+\frac{2 \epsilon}{1-a \tau}\left[\frac{-e^{-2 a t}+1}{2 a}+\frac{e^{-\left(a+\tau^{-1}\right) t}-1}{a+\tau^{-1}}\right]+\frac{2 \epsilon \tau}{\left(1+\left|a_{0}\right| \tau\right)(1+a \tau)}\left(1-e^{-\left(a+\tau^{-1}\right) t}\right),
$$

where the last term in the rhs of (2.12) originates in the cross correlation (2.10). For times $t \gg a^{-1}$, the quantity $\left\langle h^{2}(t)\right\rangle$ becomes time independent,

$$
\left.\left\langle h^{2}(t)\right\rangle \simeq\left\langle h^{2}(\infty)\right\rangle \equiv\left\langle h^{2}\right\rangle=\epsilon \mid \frac{1}{\left|a_{0}\right|\left(1+\left|a_{0}\right| \tau\right)}+\frac{1}{a(1+a \tau)}+\frac{2 \tau}{\left(1+\left|a_{0}\right| \tau\right)(1+a \tau)}\right),
$$

so that, inverting Eq. (2.8),

$$
t=\frac{1}{2 a} \ln \frac{I}{h^{2}},
$$

and we easily derive an explicit equation for the generating function

$W(\lambda)=\left\langle e^{-\lambda t}\right\rangle=\Gamma\left(\frac{\lambda}{2 a}+1\right)\left(\frac{I}{\left\langle h^{2}\right\rangle}\right)^{-\lambda / 2 a}$.

This result is formally equivalent to the corresponding one $^{3}$ for $\tau=0$ but now with $\left\langle h^{2}\right\rangle$ given by (2.13). Since the variance of the passage-time distribution $\left\langle(\Delta t)^{2}\right\rangle$ is independent of $\left\langle h^{2}\right\rangle$, it is also independent of $\tau$. For the mean first passage time we obtain

$\langle t\rangle-\langle t\rangle_{\tau=0}=\frac{1}{2 a} \ln \left(1+\frac{\left|a_{0}\right| a \tau^{2}}{1+\left(a+\left|a_{0}\right|\right) \tau}\right) \equiv \Delta$, where the white noise result is ${ }^{3}$

$$
\langle t\rangle_{\tau=0}=\frac{1}{2 a} \ln \frac{I}{\epsilon\left(\left|a_{0}\right|^{-1}+a^{-1}\right)}-\frac{1}{2 a} \psi(1)
$$

and $\psi(1)$ is the digamma function. ${ }^{15}$ We note that the correction to the white noise result is positive and independent of $\epsilon$. Its magnitude depends on $\tau$ but it is generally small in comparison with $\langle t\rangle_{\tau=0}$ for small enough noise intensity $\epsilon$. In the limit of small correlation time of the noise, $a \tau<<1$, we obtain from (2.13)

$$
\left\langle h^{2}\right\rangle=\left\langle h^{2}\right\rangle_{\tau=0}-\epsilon \tau^{2}\left(a-a_{0}\right)+\mathcal{O}\left(\tau^{3}\right),
$$

so that

$$
\langle t\rangle=\langle t\rangle_{\tau=0}+\frac{\left|a_{0}\right| \tau^{2}}{2}+\mathcal{O}\left(\tau^{3}\right)
$$

Thus the first correction to the white noise limit is of or- 
der $\tau^{2}$. The vanishing of a term linear in $\tau$ is a consequence of the presence of the cross correlation (2.10). If they were neglected, a correction of order $\tau$ would appear. This specific feature will also apply to the more general cases considered below.

\section{FAST SWEEPING}

We consider now situations of fast sweeping. This means that $I(t)$ attains a prescribed value at times typi- cally much larger than the time $t_{1}$ at which the pump parameter reaches a fixed final value $a$. A straightforward calculation yields a result for $\left\langle h^{2}(t)\right\rangle$ which contains time-independent terms and terms proportional to $\exp \left[-2 a\left(t-t_{1}\right)\right]$ or proportional to $\exp \left[-\left(a+\tau^{-1}\right)\left(t-t_{1}\right)\right]$. The time-dependent terms can be neglected for times of interest $t-t_{1} \gg a^{-1}$. This is the same condition as for the instantaneous switching but with an origin of time shifted to $t_{1}$. In this case $\left\langle h^{2}(t)\right\rangle \simeq\left\langle h^{2}(\infty)\right\rangle \equiv\left\langle h^{2}\right\rangle$ is explicitly written as

$$
\begin{aligned}
\left\langle h^{2}\right\rangle= & \frac{\epsilon}{\left|a_{0}\right|\left(1+\left|a_{0}\right| \tau\right)}+\epsilon\left[\Phi\left(x_{1}\right)-\Phi\left(x_{0}\right)\right] e^{x_{0}^{2}}\left[\Phi\left(\left|y_{0}\right|\right) \frac{\pi}{\alpha \tau} e^{y_{0}^{2}}+\frac{1}{1+\left|a_{0}\right| \tau}\left(\frac{2 \pi}{\alpha}\right)^{1 / 2}\right] \\
& +\frac{\epsilon}{\tau}\left[\frac{2 \pi}{\alpha}\right]^{1 / 2} e^{y_{0}^{2}} \int_{0}^{t_{1}} \Phi\left(y\left(t^{\prime}\right)\right) \exp \left[-\left[\frac{\alpha}{2} t^{\prime 2}+\left(a_{0}+\tau^{-1}\right) t^{\prime}\right]\right] d t^{\prime} \\
& +\frac{\epsilon}{1+a \tau}\left\{\left[\frac{2 \pi}{\alpha}\right]^{1 / 2} e^{y_{0}^{2}} \exp \left[-\left[\frac{\alpha}{2} t_{1}^{2}+\left(a_{0}+\tau^{-1}\right) t_{1}\right]\right]\left[\Phi\left(y_{1}\right)-\Phi\left(y_{0}\right)\right]\right. \\
& \left.+\frac{2 \tau}{1+\left|a_{0}\right| \tau} \exp \left[-\left[\frac{\alpha}{2} t_{1}^{2}+\left(a_{0}+\tau^{-1}\right) t_{1}\right]\right]+\frac{1}{a} \exp \left[-\left(\alpha t_{1}^{2}+2 a_{0} t_{1}\right)\right]\right\},
\end{aligned}
$$

where $t_{1}=\left(a-a_{0}\right) / \alpha, \Phi$ is the error function,

$$
\begin{aligned}
& x_{0}=\frac{1+a_{0} \tau}{(2 \alpha)^{1 / 2} \tau}, \quad x_{1}=\frac{1+a \tau}{(2 \alpha)^{1 / 2} \tau}, \\
& y(t)=\frac{-1+a(t) \tau}{(2 \alpha)^{1 / 2} \tau}, \quad y_{0}=\frac{-1+a_{0} \tau}{(2 \alpha)^{1 / 2} \tau}, \quad y_{1}=y\left(t_{1}\right) .
\end{aligned}
$$

Under the time-independent approximation (3.1) for $\left\langle h^{2}\right\rangle$, the calculation of the passage-time statistics is completely similar to the corresponding one in the limit $\tau=0$ (Ref. 3). The expressions for the generating function $W(\lambda)$, mean first passage time $\langle t\rangle$, and variance of the passage-time distribution $\left\langle(\Delta t)^{2}\right\rangle$ in terms of $\left\langle h^{2}\right\rangle$ are then

$$
\begin{aligned}
& W(\lambda)=\left[\frac{I}{\left\langle h^{2}\right\rangle}\right]^{-\lambda /(2 a)} \exp \left[-\lambda \frac{\left(a+\left|a_{0}\right|\right)^{2}}{2 a \alpha}\right] \Gamma\left[\frac{\lambda}{2 a}+1\right], \\
& \langle t\rangle=\frac{1}{2 a} \ln \frac{I}{\left\langle h^{2}\right\rangle}+\frac{\left(a+\left|a_{0}\right|\right)^{2}}{2 a \alpha}-\frac{\psi(1)}{2 a}, \\
& \left\langle(\Delta t)^{2}\right\rangle=\frac{1}{4 a^{2}} \psi^{\prime}(1) .
\end{aligned}
$$

The consistency of this results with the assumption $t-t_{1} \gg a^{-1}$ is known to require, already in the white noise limit, a small noise intensity condition $\epsilon<<a$ and also the fast sweeping condition $a \lesssim \alpha^{1 / 2}$. We will always assume $\left|a_{0}\right| \sim a$. Within these limitations, the presence of the parameter $\tau \neq 0$ allows us to consider different situations. The importance of colored noise is measured in terms of the time scale $\alpha^{-1 / 2}$ fixed by the sweeping rate. The situation $\alpha^{1 / 2} \tau<<1$ (and $a$ fortiori $a \tau<<1$ ) corresponds to the vicinity of the white noise limit, while for $\alpha^{1 / 2} \tau \gg 1$ the importance of the colored noise should be more noticeable. We next consider these two situations separately.

In the limit $\alpha^{1 / 2} \tau<<1,(3.1)$ can be expanded around the white noise limit. We obtain (see Appendix A)

$$
\left\langle h^{2}\right\rangle=\frac{\epsilon}{\left|a_{0}\right|}+\epsilon a^{-1} e^{-\left(a^{2}-a_{0}^{2}\right) / \alpha}+\epsilon \alpha^{-1 / 2} \pi^{1 / 2} e^{a_{0}^{2} / \alpha}\left[\Phi\left(a \alpha^{-1 / 2}\right)+\Phi\left(\left|a_{0}\right| \alpha^{-1 / 2}\right)\right]\left[1-\frac{1}{2}\left(\alpha^{1 / 2} \tau\right)^{2}+\mathcal{O}\left(\left(\alpha^{1 / 2} \tau\right)^{3}\right)\right]
$$

and substituting in (3.5)

$$
\begin{aligned}
\langle t\rangle & =\langle t\rangle_{\tau=0}+\frac{1}{2 a} \ln \frac{\left\langle h^{2}\right\rangle_{\tau=0}}{\left\langle h^{2}\right\rangle} \\
& \simeq\langle t\rangle_{\tau=0}+\left(\alpha^{1 / 2} \tau\right)^{2} \frac{\pi^{1 / 2} e^{a_{0}^{2} / \alpha}\left[\Phi\left(a \alpha^{-1 / 2}\right)+\Phi\left(\left|a_{0}\right| \alpha^{-1 / 2}\right)\right]}{4 a \alpha^{1 / 2} \epsilon^{-1}\left\langle h^{2}\right\rangle_{\tau=0}}
\end{aligned}
$$


where

$$
\langle t\rangle_{\tau=0}=\frac{1}{2 a} \ln \frac{I}{\left\langle h^{2}\right\rangle_{\tau=0}}+\frac{\left(a+\left|a_{0}\right|\right)^{2}}{2 a \alpha}-\frac{\psi(1)}{2 a} .
$$

Therefore the mean first passage time increases, to lowest order in $\alpha^{1 / 2} \tau$, in a quantity which is independent of $\epsilon$ as in (2.19). Also as it happened in the instantaneous switch, the first correction is already of order $\tau^{2}$.

The limit of large correlation time $\alpha^{1 / 2} \tau \gg 1$ admits several particular cases, because $\left\langle h^{2}\right\rangle$ in (3.1) does not only depend on $\tau$ through $\alpha^{1 / 2} \tau$. Two interesting cases appear when comparing the time scale $\tau$ of the noise with the time scale $\alpha^{-1}$ fixed by the finite value of the pump parameter. When these two time scales are comparable, $a \tau \sim 1$, we have that $\alpha^{1 / 2} \gg a$ and we are close to the instantaneous switch discussed in Sec. II. The new case of interest in which colored noise is important is when $a \tau \gg 1$. Given our general restriction of fast sweeping $a \lesssim \alpha^{1 / 2}$, this case corresponds to comparable time scales fixed by $a$ and $\alpha^{1 / 2}$ and also to switching far above threshold in relation with the inverse of the correlation time of the noise $(a \tau \gg>1)$.

Close to the instantaneous switch, $\alpha^{1 / 2}>a$, and with no restriction on $a \tau$, one can obtain from (3.1) (Appendix A)

$$
\begin{aligned}
\left\langle h^{2}\right\rangle \simeq\left\langle h^{2}\right\rangle_{\alpha \rightarrow \infty}+ & \epsilon a^{-1}\left[\left(a+\left|a_{0}\right|\right) \alpha^{-1 / 2}\right]^{2} \\
& \times \frac{1+\left(a+\left|a_{0}\right|\right) \tau}{(1+a \tau)\left(1+\left|a_{0}\right| \tau\right)},
\end{aligned}
$$

where $\left\langle h^{2}\right\rangle_{\alpha \rightarrow \infty}$ corresponds to the instantaneous switch and it is given by (2.13). Replacing (3.10) in (3.5) we obtain to lowest order in $a \alpha^{-1 / 2}$,

$$
\langle t\rangle=\langle t\rangle_{\alpha \rightarrow \infty}+\frac{1}{2}\left(a+\left|a_{0}\right|\right) \alpha^{-1},
$$

where $\langle t\rangle_{\alpha \rightarrow \infty}$ is given in (2.16). The result (3.11) indicates that close to the instantaneous switch, the first correction to $\langle t\rangle$ is independent of $\tau$. Indeed, $\langle t\rangle-\langle t\rangle_{\alpha \rightarrow \infty}$ given by (3.11) coincides with the result obtained for $\tau=0$ in Ref. 3. Equation (3.11) combined with (2.16) gives rise to an interesting result concerning the effect of $\tau$ in this limit $\alpha^{1 / 2} \tau \gg 1$. Indeed, from (3.11)

$$
\langle t\rangle-\langle t\rangle_{\alpha \rightarrow \infty}=\langle t\rangle_{\tau=0}-\langle t\rangle_{\tau=0, \alpha \rightarrow \infty},
$$

so that

$$
\langle t\rangle-\langle t\rangle_{\tau=0}=\langle t\rangle_{\alpha \rightarrow \infty}-\langle t\rangle_{\tau=0, \alpha \rightarrow \infty}=\Delta,
$$

whose explicit expression is given in (2.16). Therefore, in the range of parameters $\alpha^{1 / 2} \tau \gg 1, a \tau \sim 1$, the difference between $\langle t\rangle$ and its white-noise limit turns out to be $\alpha$ independent and it may be consequently obtained from the analysis of the instantaneous switch.

The situation of large correlation time $\alpha^{1 / 2} \tau \gg 1$ with $a \tau \gg 1$ can also be analyzed from (3.1). In this limit (Appendix A) we obtain

$$
\left\langle h^{2}\right\rangle=\epsilon\left(\alpha^{1 / 2} \tau\right)^{-1} A
$$

where $A$ is a quantity given in Appendix A which is independent of $\tau$ and $\epsilon$. Substituting (3.14) in (3.5) we ob-

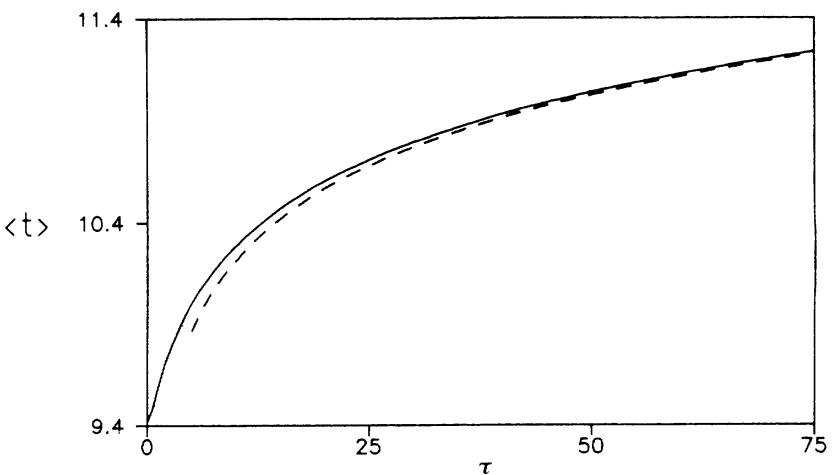

FIG. 1. $\langle t\rangle$ vs $\tau$ as obtained from (3.1) and (3.5). Values of the parameters: $a=\left|a_{0}\right|=1, \alpha=4, \epsilon / I=10^{-8}$. For $\tau=0$, (3.9) gives $\langle t\rangle_{\tau=0}=9.42$. Dashed line corresponds to the approximation (3.15).

tain that for small $\epsilon$ and $\alpha^{1 / 2} \tau \gg 1$ the dominant contribution is given by

$$
\langle t\rangle=\frac{1}{2 a} \ln \frac{I \alpha^{1 / 2} \tau}{\epsilon} .
$$

This result does not correspond to a correction to any previously known result. It gives a quantity diverging logarithmically with $\alpha^{1 / 2} \tau$ and it identifies a domain of parameters in which the correlation time of the noise has important quantitative consequences on the value of $\langle t\rangle$.

Our results of this section are better summarized in Figs. 1 and 2 . In both figures we show the mean first passage time $\langle t\rangle$ as calculated from (3.5) and the general result (3.1) for $\left\langle h^{2}\right\rangle$. Figure 1 shows the monotonous growth of $\langle t\rangle$ with the correlation time of the noise. Figure 2 shows that $\langle t\rangle$ is a monotonous decreasing function of $\alpha$. Figure 1 shows how our general result connects the limits $\alpha^{1 / 2} \tau \gg>1$ and $\alpha^{1 / 2} \tau<<1$ considered above in detail. For $\alpha^{1 / 2} \tau \gg 1$ Fig. 1 refers to the case $a \tau \gg 1$, while Fig. 2 shows the result for $a \tau \sim 1$ in the domain from $\alpha^{1 / 2} \tau \sim 1$ to $\alpha^{1 / 2} \tau \gg>1$. The value of $\langle t\rangle$ in Fig. 1 is given for small $\tau$ by $(3.8)-(3.9)$ and for large $\tau$

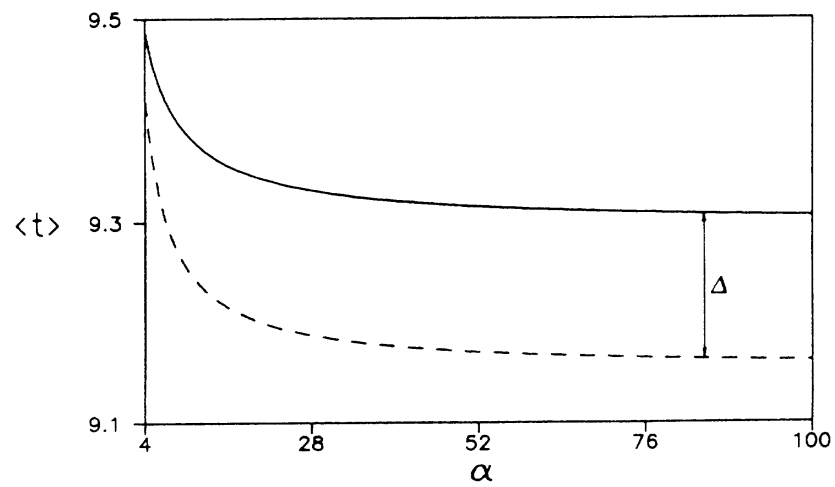

FIG. 2. $\langle t\rangle$ vs $\alpha$ as obtained from (3.1) and (3.5). Values of the parameters: $a=\left|a_{0}\right|=1, \epsilon / I=10^{-8}$. Solid line corresponds to $\tau=1$ and dashed line to $\tau=0$. For these parameters $\Delta=\frac{1}{2} \ln \frac{4}{3}=0.14$. For $\alpha=100$, we find $\langle t\rangle_{\tau=0}=9.162$, $\langle t\rangle_{\tau=1}=9.306$. 
by (3.15). The dashed line identifies the domain validity of the asymptotic expression (3.15). It is shown that (3.15) gives a simple and accurate result for the domain of parameters in which the role of the correlation time of the noise is important. Figure 2 makes clear the contents of (3.13) for $\alpha^{1 / 2} \tau \gg 1, a \tau \sim 1$ : for large enough $\alpha$, the curves associated with $\tau=0$ and $\tau \neq 0$ are essentially parallel and separated by a quantity $\Delta$ given by (2.16).

\section{SLOW SWEEPING}

The general condition of slow sweeping is that all times of interest are smaller than $t_{1}$. This corresponds to taking $a \rightarrow \infty$, so that this parameter no longer appears in the calculations. In other words, we do not consider any more the time scale associated with $a$, as we did in the case of fast sweeping. As in Sec. III, we consider the two limiting cases of small $\left(\alpha^{1 / 2} \tau<<1\right)$ and large $\left(\alpha^{1 / 2} \tau>>1\right)$ correlation time of the noise. In the case of slow sweeping, however, a time-independent approximation for $\left\langle h^{2}(t)\right\rangle$, valid for all values of $\tau$, is not very useful in order to obtain analytical results. As a consequence, we consider from the beginning separately the two cases $\alpha^{1 / 2} \tau<<1$ and $\alpha^{1 / 2} \tau \gg 1$. In any case, once a timeindependent approximation for $\left\langle h^{2}\right\rangle$ is obtained, the calculation is exactly the same as for $\tau=0$, yielding analogous formal expressions in terms of $\left\langle h^{2}\right\rangle$ (Ref. 3):

$$
\begin{aligned}
& W(\lambda)=\Gamma\left[\frac{\lambda}{2} \alpha^{-1 / 2} T^{-1 / 2}+1\right] \exp \left\{-\lambda\left[\frac{\left|a_{0}\right|}{\alpha}+\alpha^{-1 / 2} T^{1 / 2}\left[1+\frac{a_{0}^{2}}{2 \alpha T}\right]\right]\right\} \\
& \langle t\rangle=\frac{\left|a_{0}\right|}{\alpha}+\alpha^{-1 / 2} T^{1 / 2}+\frac{1}{2} T^{-1 / 2}\left[a_{0}^{2} \alpha^{-3 / 2}-\psi(1) \alpha^{-1 / 2}\right], \\
& \left\langle(\Delta t)^{2}\right\rangle=\frac{1}{4 \alpha} T^{-1} \psi^{\prime}(1),
\end{aligned}
$$

where

$$
T \equiv \ln \left(I /\left\langle h^{2}\right\rangle\right) .
$$

These results are valid in the weak-noise assumption $\epsilon<<\alpha^{1 / 2}$ and for $\left|a_{0}\right| \sim \alpha^{1 / 2}$.

In the case of small correlation time of the noise $\left(\alpha^{1 / 2} \tau<<1\right)$, the integration of (2.9) can be approximated by a timeindependent quantity for times of interest $t-\bar{t} \gg \alpha^{-1 / 2}$ as (Appendix B)

$$
\left\langle h^{2}\right\rangle=\frac{\epsilon}{\left|a_{0}\right|}+\epsilon\left(\frac{\pi}{\alpha}\right)^{1 / 2} e^{a_{0}^{2} / \alpha}\left[\Phi\left(\left|a_{0}\right| \alpha^{-1 / 2}\right)+1\right]\left[1-\frac{1}{2}\left(\alpha^{1 / 2} \tau\right)^{2}+\mathcal{O}\left(\left(\alpha^{1 / 2} \tau\right)^{3}\right)\right]
$$

In the same way that in the limit $\tau=0$, the requirement $t-\bar{t} \gg \alpha^{-1 / 2}$ is satisfied for $\epsilon<<\alpha^{-1 / 2}$ and $\left|a_{0}\right| \sim \alpha^{1 / 2}$. The correction to the white-noise limit is exactly of the same form as in (3.7). Also in the same way as in previous cases, the first correction is already of order $\tau^{2}$.

The mean first passage time $\langle t\rangle$ can be calculated replacing (4.5) in (4.2). Even if in (4.5) the correction to the whitenoise limit is formally identical to the corresponding one in (3.7), the actual correction to $\langle t\rangle$ is rather different because of the different dependence of $\langle t\rangle$ on $\left\langle h^{2}\right\rangle$. Writing $T=T_{\tau=0}+\ln \left(\left\langle h^{2}\right\rangle_{\tau=0} /\left\langle h^{2}\right\rangle\right)$ and taking into account that both $T$ and $T_{\tau=0}$ are large quantities of order $\ln \left(\epsilon^{-1} I\right)$, the mean first passage time $\langle t\rangle$ to lowest order in $\tau$ is easily obtained as

$$
\langle t\rangle \simeq\langle t\rangle_{\tau=0}+\left(\alpha^{1 / 2} \tau\right)^{2} \frac{1}{4\left(\alpha T_{\tau=0}\right)^{1 / 2}} \frac{\pi^{1 / 2} e^{a_{0}^{2} / \alpha}\left[\Phi\left(\left|a_{0}\right| \alpha^{-1 / 2}\right)+1\right]}{\left|a_{0}\right|^{-1} \alpha^{1 / 2}+\pi^{1 / 2} e^{a_{0}^{2} / \alpha}\left[\Phi\left(\left|a_{0}\right| \alpha^{-1 / 2}\right)+1\right]}
$$

A main difference between (4.6) and (3.8) is that in the present case of slow sweeping the correction to the white-noise limit depends on $\epsilon$ through $T_{\tau=0}^{-1 / 2} \sim\left(\ln \epsilon^{-1} I\right)^{-1 / 2}$. This dependence implies that the correction of order $\left(\alpha^{1 / 2} \tau\right)^{2}$ is weighted in the considered case of small noise by a rather small quantity. The consequence is that the effect of the finite correlation time of the noise is here much smaller than in the case of fast sweeping. However, the variance $\left\langle(\Delta t)^{2}\right\rangle$ that was independent of $\tau$ for fast sweeping becomes now $\tau$-dependent. Since $\left\langle h^{2}\right\rangle$ decreases with $\tau$ with respect to the white-noise limit, $T$ increases and $\left\langle(\Delta t)^{2}\right\rangle(4.3)$ is reduced with respect to the white-noise limit

$$
\left\langle(\Delta t)^{2}\right\rangle=\left\langle(\Delta t)^{2}\right\rangle_{\tau=0}-\frac{\left(\alpha^{1 / 2} \tau\right)^{2} \psi^{\prime}(1)}{8 \alpha T_{\tau=0}^{2}} \frac{\pi^{1 / 2} e^{a_{0}^{2} / \alpha}\left[\Phi\left(\left|a_{0}\right| \alpha^{-1 / 2}\right)+1\right]}{\left|a_{0}\right|^{-1} \alpha^{1 / 2}+\pi^{1 / 2} e^{a_{0}^{2} / \alpha}\left[\Phi\left(\left|a_{0}\right| \alpha^{-1 / 2}\right)+1\right]}
$$


Let us finally consider the case of large correlation time $\tau, \alpha^{1 / 2} \tau \gg 1$. Again, for times of interest $t-\bar{t} \gg \alpha^{-1 / 2}$, a time-independent approximation for $\left\langle h^{2}(t)\right\rangle$ can be obtained (Appendix B):

$$
\left\langle h^{2}\right\rangle=\epsilon\left(\alpha^{1 / 2} \tau\right)^{-1} B,
$$

where $B$ is a quantity given in Appendix $\mathbf{B}$ and which is also independent of $\tau$ and $\epsilon$. The result (4.8) is thus reminiscent of (3.14). Again a different dependence of $\langle t\rangle$ on $\tau$ with respect to the fast sweeping case appears because of the different dependence on $\left\langle h^{2}\right\rangle$ implied by (4.2). Substituting (4.8) in (4.2), and for small $\epsilon$ and $\alpha^{1 / 2} \tau \gg>1$, we find

$$
\langle t\rangle-\frac{\left|a_{0}\right|}{\alpha} \simeq \alpha^{-1 / 2}\left[\ln \left[\frac{I \alpha^{1 / 2} \tau}{\epsilon}\right]\right]^{1 / 2} .
$$

The square-root law in (4.9) is characteristic of the slowsweeping situation. As in (3.15), this asymptotic result does not correspond to a correction to any known result. It gives the asymptotic form of $\langle t\rangle$ in the domain of parameter in which the correlation time of the noise is important in situations of slow sweeping. Also, as in (3.15), it gives a divergence of $\langle t\rangle$ with $\tau \rightarrow \infty$, but it is here weaker due to the square-root law.

As a general conclusion, we observe that a finite correlation time of the noise has the same qualitative implications for fast and slow sweeping, but its effect seems to be quantitatively more important for fast sweeping.

\section{ACKNOWLEDGMENTS}

Financial support from the Dirección General de Investigación Cientifica y Técnica (Spain), Project Nos. PB86-0534 and PB87-0014 is acknowledged. Thanks are also given to L. Ramirez de la Piscina for his help in numerical calculations.

\section{APPENDIX A: FAST SWEEPING $\left(\epsilon<<a \lesssim \alpha^{1 / 2}\right)$}

In this appendix we derive the three limiting situations of (3.1) considered in Sec. III.

$$
\text { 1. } \alpha^{1 / 2} \tau<<1
$$

The first and last terms in (3.1), with $t_{1}=\left(a-a_{0}\right) / \alpha$, admit a simple treatment in powers of $\alpha^{1 / 2} \tau$,

$$
\begin{aligned}
& \frac{\epsilon}{\left|a_{0}\right|\left(1+\left|a_{0}\right| \tau\right)}+\frac{\epsilon}{a(1+a \tau)} e^{-\left(a^{2}-a_{0}^{2}\right) / \alpha} \\
& \quad=\epsilon\left(\left|a_{0}\right|^{-1}+a^{-1} e^{-\left(a^{2}-a_{0}^{2}\right) / \alpha}\right)-\epsilon \tau\left(1+e^{-\left(a^{2}-a_{0}^{2}\right) / \alpha}\right)+\epsilon \tau^{2}\left(\left|a_{0}\right|+a e^{-\left(a^{2}-a_{0}^{2}\right) / \alpha}\right)+\mathcal{O}\left(\left(\alpha^{1 / 2} \tau\right)^{3}\right) .
\end{aligned}
$$

We next analyze the contributions to (3.1) arising from the non-Markovian correlation of the initial condition $E_{i}(0)$ and the noise [see (2.10)]. Using the expansion of the error functions $\Phi(z)$ (Ref. 16) around infinity $\left[\Phi(z) \simeq 1-e^{-z^{2}} /\left(\pi^{1 / 2} z\right)+e^{-z^{2}} /\left(2 \pi^{1 / 2} z^{3}\right)+\cdots\right]$ and substituting $x_{0}$ and $x_{1}$ from (3.2), we finally arrive at

$$
\frac{\epsilon}{1+\left|a_{0}\right| \tau}\left\{\left(\frac{2 \pi}{\alpha}\right)^{1 / 2} e^{x_{0}^{2}}\left[\Phi\left(x_{1}\right)-\Phi\left(x_{0}\right)\right]+\frac{2 \tau}{1+a \tau} \exp \left[-\left(\frac{\alpha}{2} t_{1}^{2}+\left(a_{0}+\tau^{-1}\right) t_{1}\right]\right)\right\}=2 \epsilon \tau+\mathcal{O}\left(\left(\alpha^{1 / 2} \tau\right)^{3}\right)
$$

The remaining terms in (3.1) are those containing error functions of arguments $y$. Invoking again a series development of $\Phi(z)$ around larger values of $z$, we have

$$
\begin{aligned}
& \epsilon e^{y_{0}^{2}}\left\{\frac{\pi}{\alpha \tau} \Phi\left(\left|y_{0}\right|\right) e^{x_{0}^{2}}\left[\Phi\left(x_{1}\right)-\Phi\left(x_{0}\right)\right]+\left[\frac{2 \pi}{\alpha}\right]^{1 / 2} \frac{1}{\tau} \int_{0}^{t_{1}} \Phi\left(y\left(t^{\prime}\right)\right) \exp \left[-\left[\frac{\alpha}{2} t^{\prime 2}+\left(a_{0}+\tau^{-1}\right) t^{\prime}\right]\right) d t^{\prime}\right. \\
& \left.+\left(\frac{2 \pi}{\alpha}\right)^{1 / 2} \frac{1}{1+a \tau} \exp \left[-\left(\frac{\alpha}{2} t_{1}^{2}+\left(a_{0}+\tau^{-1}\right) t_{1}\right)\right]\left[\Phi\left(y_{1}\right)-\Phi\left(y_{0}\right)\right]\right\} \\
& =\frac{\epsilon}{\tau} \frac{2 \pi}{\alpha} e^{y_{0}^{2}}\left\{( 1 - \frac { e ^ { - y _ { 0 } ^ { 2 } } } { \pi ^ { 1 / 2 } | y _ { 0 } | } + \cdots ) \int _ { 0 } ^ { t _ { 1 } } \operatorname { e x p } \left[-\left(\frac{\alpha}{2} t^{\prime 2}+\left(a_{0}+\tau^{-1}\right) t^{\prime}\right] d t^{\prime}\right.\right. \\
& -\int_{0}^{t_{1}}\left[1-\frac{e^{-y\left(t^{\prime}\right)}}{\pi^{1 / 2}\left|y\left(t^{\prime}\right)\right|}+\frac{e^{-y\left(t^{\prime}\right)}}{2 \pi^{1 / 2}\left|y\left(t^{\prime}\right)\right|^{3}}+\cdots\right) \exp \left[-\left[\frac{\alpha}{2} t^{\prime 2}+\left(a_{0}+\tau^{-1}\right) t^{\prime}\right] d t^{\prime}\right. \\
& +\frac{\epsilon}{1+a \tau}\left[\frac{2 \pi}{\alpha}\right]^{1 / 2} e^{y_{0}^{2}}\left[\frac{e^{-y_{1}^{2}}}{\pi^{1 / 2}\left|y_{1}\right|}-\frac{e^{-y_{0}^{2}}}{\pi^{1 / 2}\left|y_{0}\right|}+\cdots\right) \exp \left[-\left(\frac{\alpha}{2} t_{1}^{2}+\left(a_{0}+\tau^{-1}\right) t_{1}\right]\right] \\
& =\left[\frac{\pi}{\alpha}\right]^{1 / 2} e^{a_{0}^{2} / \alpha}\left[\Phi\left(a \alpha^{-1 / 2}\right)+\Phi\left(\left|a_{0}\right| \alpha^{-1 / 2}\right)\right]-\epsilon \tau\left(1-e^{-\left(a^{2}-a_{0}^{2}\right) / \alpha}\right) \\
& +\epsilon \tau^{2}\left[a_{0}-a e^{-\left(a^{2}-a_{0}^{2}\right) / \alpha}-\frac{(\pi \alpha)^{1 / 2}}{2} e^{a_{0}^{2} / \alpha}\left[\Phi\left(a \alpha^{-1 / 2}\right)+\Phi\left(\left|a_{0}\right| \alpha^{-1 / 2}\right)\right]\right]+\mathcal{O}\left(\left(\alpha^{1 / 2} \tau\right)^{3}\right)
\end{aligned}
$$


Adding up the results (A1)-(A3), we obtain (3.7).

$$
\text { 2. } \alpha^{1 / 2} \tau \gg 1, a \tau \sim 1
$$

The last two terms in (3.1), with $t_{1}=\left(a-a_{0}\right) / \alpha$, are simply expanded in powers of $\left(\alpha^{1 / 2} \tau\right)^{-1}$, giving

$$
\begin{aligned}
\frac{\epsilon}{1+a \tau}\left\{\frac{2 \tau}{1+\left|a_{0}\right| \tau} \exp [\right. & \left.\left.-\left[\frac{\tau\left(a^{2}-a_{0}^{2}\right)+2\left(a-a_{0}\right)}{2 \alpha \tau}\right]\right]+\frac{1}{a} e^{-\left(a^{2}-a_{0}^{2}\right) / \alpha}\right\} \\
= & \frac{\epsilon}{1+a \tau}\left[\frac{2 \tau}{1+\left|a_{0}\right| \tau}+\frac{1}{a}-\frac{a-a_{0}}{\alpha}\left[\frac{\tau\left(a+a_{0}\right)}{1+\left|a_{0}\right| \tau}+\frac{a+a_{0}}{a}+\frac{2}{1+\left|a_{0}\right| \tau}\right]+\mathcal{O}\left(\left(\alpha^{1 / 2} \tau\right)^{-4}\right)\right] .
\end{aligned}
$$

Using the expansion of $\Phi(z)$ around zero $\left[\Phi(z)=\left(2 / \pi^{1 / 2}\right)\left(z-z^{3} / 3+\cdots\right)\right]$, the contributions in (3.1) involving $\Phi(x)$ read, to second order,

$$
\epsilon\left[\Phi\left(x_{1}\right)-\Phi\left(x_{0}\right)\right] e^{x_{0}^{2}}\left[\Phi\left(\left|y_{0}\right|\right) \frac{\pi}{\alpha \tau} e^{y_{0}^{2}}+\frac{1}{1+\left|a_{0}\right| \tau}\left[\frac{2 \pi}{\alpha}\right]^{1 / 2}\right]=\frac{2 \epsilon}{\alpha} \frac{a-a_{0}}{1+\left|a_{0}\right| \tau}+\mathcal{O}\left(\left(\alpha^{1 / 2} \tau\right)^{-4}\right) .
$$

With the same criterion, we handle the remaining contributions involving $\Phi(y)$

$\epsilon\left[\frac{2 \pi}{\alpha}\right]^{1 / 2} e^{y_{0}^{2}}\left\{\frac{1}{\tau} \int_{0}^{t_{1}} \Phi\left(y\left(t^{\prime}\right)\right) \exp \left[-\left[\frac{\alpha}{2} t^{\prime 2}+\left(a_{0}+\tau^{-1}\right) t^{\prime}\right]\right] d t^{\prime}\right.$

$$
\begin{aligned}
& \left.+\frac{1}{1+a \tau} \exp \left[-\left[\frac{\alpha}{2} t_{1}^{2}+\left(a_{0}+\tau^{-1}\right) t_{1}\right]\right]\left[\Phi\left(y_{1}\right)-\Phi\left(y_{0}\right)\right]\right\} \\
& =\frac{\epsilon}{\tau}\left[\frac{\pi}{2 \alpha}\right]^{1 / 2} \frac{\pi}{\alpha} e^{2 y_{0}^{2}} e^{2\left(a_{0} \tau-1\right) /\left(\alpha \tau^{2}\right)} \int_{y_{0}}^{y_{1}} e^{-2 \sqrt{2} y^{\prime} / \alpha^{1 / 2} \tau} \Phi\left(y^{\prime}\right) \Phi^{\prime}\left(y^{\prime}\right) d y^{\prime} \\
& +\frac{2 \epsilon}{\alpha} \frac{a-a_{0}}{1+a \tau}+\mathcal{O}\left(\left(\alpha^{1 / 2} \tau\right)^{-4}\right) \\
& =\frac{\epsilon \pi}{\alpha \tau} e^{\left[\left(a_{0} \tau\right)^{2}-1\right] /\left(\alpha \tau^{2}\right)}\left\{\frac{1}{2}\left[\Phi^{2}\left(y_{1}\right)-\Phi^{2}\left(y_{0}\right)\right]+\frac{\sqrt{2}}{\alpha^{1 / 2} \tau}\left[\Phi\left(y_{1}\right) \Phi^{\prime}\left(y_{1}\right)-\Phi\left(y_{0}\right) \Phi^{\prime}\left(y_{0}\right)\right.\right. \\
& \left.-\left(\frac{2}{\pi}\right)^{1 / 2}\left[\Phi\left(\sqrt{2} y_{1}\right)-\Phi\left(\sqrt{2} y_{0}\right)\right]\right] \\
& \left.-\frac{1}{(2 \pi)^{1 / 2}} \frac{1}{\left(\alpha^{1 / 2} \tau\right)^{2}}\left[\Phi^{\prime}\left(\sqrt{2} y_{1}\right)-\Phi^{\prime}\left(\sqrt{2} y_{0}\right)\right]+\mathcal{O}\left(\left(\alpha^{1 / 2} \tau\right)^{-3}\right)\right\} \\
& +\frac{2 \epsilon}{\alpha} \frac{a-a_{0}}{1+a \tau}+\mathcal{O}\left(\left(\alpha^{1 / 2} \tau\right)^{-4}\right) \\
& =\frac{2 \epsilon}{\alpha} \frac{a-a_{0}}{1+a \tau}+\mathcal{O}\left(\left(\alpha^{1 / 2} \tau\right)^{-4}\right) \text {, }
\end{aligned}
$$

with $y^{\prime}=y\left(t^{\prime}\right)$. Adding the results (A4)-(A6) together with the first term in (3.1), we obtain (3.10).

3. $\alpha^{1 / 2} \tau \gg 1, a \tau \gg 1$

The first and last two terms in (3.1), with $t_{1}=\left(a-a_{0}\right) / \alpha$, are again expanded in powers of $\left(\alpha^{1 / 2} \tau\right)^{-1}$, giving

$$
\begin{aligned}
\frac{\epsilon}{\left|a_{0}\right|\left(1+\left|a_{0}\right| \tau\right)}+\frac{\epsilon}{a(1+a \tau)}\left\{\frac{2 \tau}{1+\left|a_{0}\right| \tau} \exp [\right. & \left.\left.-\left[\frac{\alpha}{2} t_{1}^{2}+\left(a_{0}+\tau^{-1}\right) t_{1}\right]\right]+\frac{1}{a} \exp \left[-\left(\alpha t_{1}^{2}+2 a_{0} t_{1}\right)\right]\right\} \\
& =\frac{\epsilon}{\tau}\left[\frac{1}{a_{0}^{2}}+\frac{1}{a^{2}} e^{-\left(a^{2}-a_{0}^{2}\right) / \alpha}+\frac{2}{\left|a_{0}\right| a} e^{-\left(a^{2}-a_{0}^{2}\right) / 2 \alpha}\right]+\mathcal{O}\left(\left(\alpha^{1 / 2} \tau\right)^{-2}\right) .
\end{aligned}
$$

The remaining contributions containing the error functions are conveniently treated using the same steps as for the integral term in (A6) and an expansion of $\Phi\left(z_{0}+z\right)$ around $z_{0}\left(\Phi\left(z_{0}+z\right)=\Phi\left(z_{0}\right)+e^{-z_{0}^{2}}\left[z+\mathcal{O}\left(z^{2}\right)\right]\right)$ 


$$
\begin{gathered}
\epsilon\left[\Phi\left(x_{1}\right)-\Phi\left(x_{0}\right)\right] e^{x_{0}^{2}}\left[\Phi\left(\left|y_{0}\right|\right) \frac{\pi}{\alpha \tau} e^{y_{0}^{2}}+\frac{1}{1+\left|a_{0}\right| \tau}\left[\frac{2 \pi}{\alpha}\right]^{1 / 2}\right] \\
+\epsilon\left(\frac{2 \pi}{\alpha}\right) 1 / 2 e^{y_{0}^{2}}\left\{\frac{1}{\tau} \int_{0}^{t_{1}} \Phi\left(y\left(t^{\prime}\right)\right) \exp \left[-\left(\frac{\alpha}{2} t^{\prime 2}+\left(a_{0}+\tau^{-1}\right) t^{\prime}\right]\right] d t^{\prime}\right. \\
\left.\quad+\frac{1}{1+a \tau} \exp \left[-\left[\frac{\alpha}{2} t_{1}^{2}+\left(a_{0}+\tau^{-1}\right) t_{1}\right]\right]\left[\Phi\left(y_{1}\right)-\Phi\left(y_{0}\right)\right]\right\} \\
=\frac{\epsilon}{\alpha^{1 / 2} \tau}\left[\Phi\left(a(2 \alpha)^{-1 / 2}\right)+\Phi\left(\left|a_{0}\right|(2 \alpha)^{-1 / 2}\right)\right] \\
\times\left[\frac{\pi}{2 \alpha^{1 / 2}} e^{a_{0}^{2} / \alpha}\left[\Phi\left(a(2 \alpha)^{-1 / 2}\right)+\Phi\left(\left|a_{0}\right|(2 \alpha)^{-1 / 2}\right)\right]+\frac{(2 \pi)^{1 / 2}}{\left|a_{0}\right|} e^{a_{0}^{2} / 2 \alpha}\right. \\
\left.\quad+\frac{(2 \pi)^{1 / 2}}{a} \exp \left(\frac{a_{0}^{2}}{\alpha}-\frac{a^{2}}{2 \alpha}\right]\right]+\mathcal{O}\left(\left(\alpha^{1 / 2} \tau\right)^{-2}\right) .
\end{gathered}
$$

Adding up the results (A7) and (A8), we obtain (3.14) with $A$ given by

$$
\begin{aligned}
A= & \frac{\alpha^{1 / 2}}{a_{0}^{2}}+\left[\frac{2}{\left|a_{0}\right|}+\frac{2}{a} e^{-\left(a^{2}-a_{0}^{2}\right) / 2 \alpha}+\left[\frac{\pi}{2 \alpha}\right]^{1 / 2} e^{a_{0}^{2} / 2 \alpha}\left[\Phi\left(a(2 \alpha)^{-1 / 2}\right)+\Phi\left(\left|a_{0}\right|(2 \alpha)^{-1 / 2}\right)\right]\right] \\
& \times\left(\frac{\pi}{2}\right]^{1 / 2} e^{a_{0}^{2} / 2 \alpha}\left[\Phi\left(a(2 \alpha)^{-1 / 2}\right)+\Phi\left(\left|a_{0}\right|(2 \alpha)^{-1 / 2}\right)\right] \\
& +\left(\frac{2}{\left|a_{0}\right|}+\frac{1}{a} e^{-\left(a^{2}-a_{0}^{2}\right) / 2 \alpha}\right] \frac{\alpha^{1 / 2}}{a} e^{-\left(a^{2}-a_{0}^{2}\right) / 2 \alpha}+\mathcal{O}\left(\left(\alpha^{1 / 2} \tau\right)^{-1}\right) .
\end{aligned}
$$

\section{APPENDIX B: SLOW SWEEPING

$$
\left(\epsilon<\alpha^{1 / 2}<<\right)
$$

In this appendix we derive, starting from (2.9), the two time-independent approximations for $\left\langle h^{2}\right\rangle$ considered in Sec. IV. Integrating (2.9), we obtain

$$
\begin{aligned}
\left\langle h^{2}(t)\right\rangle= & \left\langle h^{2}(0)\right\rangle+\epsilon\left[\Phi\left(x_{1}\right)-\Phi\left(x_{0}\right)\right] e^{x_{0}^{2}}\left[\Phi\left(\left|y_{0}\right|\right) \frac{\pi}{\alpha \tau} e^{y_{0}^{2}}+\frac{1}{1+\left|a_{0}\right| \tau}\left(\frac{2 \pi}{\alpha}\right)^{1 / 2}\right] \\
& +\frac{\epsilon}{\tau}\left(\frac{2 \pi}{\alpha}\right)^{1 / 2} e^{y_{0}^{2}} \int_{0}^{t} \Phi\left(y\left(t^{\prime}\right)\right) \exp \left[-\left[\frac{\alpha}{2} t^{\prime 2}+\left(a_{0}+\tau^{-1}\right) t^{\prime}\right]\right] d t^{\prime},
\end{aligned}
$$

where $x_{0}, x_{1}, y_{0}, y(t)$ are defined in (3.2) and (3.3), and $x(t)=[1+a(t) \tau] /\left[(2 \alpha)^{1 / 2} \tau\right]$.

$$
\text { 1. } \alpha^{1 / 2} \tau<<1
$$

The first term $\left\langle h^{2}(0)\right\rangle$ may be expanded in powers of $\alpha^{1 / 2} \tau$, giving

$$
\epsilon\left[\left|a_{0}\right|^{-1}-\tau+\tau^{2}\left|a_{0}\right|+\mathcal{O}\left(\left(\alpha^{1 / 2} \tau\right)^{3}\right)\right] \text {. }
$$

The term arising from the cross correlation (2.10) may be handled using an expansion of the error functions $\Phi(z)$ (Ref. 16) around infinity (see Appendix A). For times of interest $\left(t-\bar{t} \gg \alpha^{-1 / 2}\right)$, we can take $\Phi(x(t))=1$ so that

$$
\frac{\epsilon}{1+\left|a_{0}\right| \tau}\left[\frac{2 \pi}{\alpha}\right] e^{x_{0}^{2}}\left[\Phi(x(t))-1-\frac{e^{-x_{0}^{2}}(2 \alpha)^{1 / 2} \tau}{\pi^{1 / 2}\left(1+a_{0} \tau\right)}+\cdots\right)=2 \epsilon \tau+\mathcal{O}\left(\left(\alpha^{1 / 2} \tau\right)^{3}\right)
$$

The remaining terms are those containing error functions of arguments $y$. Using again a series development of $\Phi(z)$ around infinity, and considering that $y\left(t^{\prime}\right)$ changes sign for $t=t_{s}\left[t_{s}=\left(1+\left|a_{0}\right| \tau\right) / \alpha \tau\right]$, 


$$
\begin{aligned}
& \frac{\epsilon}{\tau} e^{y_{0}^{2}}\left\{\frac{\pi}{\alpha} \Phi\left(\left|y_{0}\right|\right) e^{x_{0}^{2}}\left[\Phi\left(x_{1}\right)-\Phi\left(x_{0}\right)\right]+\left(\frac{2 \pi}{\alpha}\right]^{1 / 2} \int_{0}^{t} \Phi\left(y\left(t^{\prime}\right)\right) \exp \left[-\left[\frac{\alpha}{2} t^{\prime 2}+\left(a_{0}+\tau^{-1}\right) t^{\prime}\right]\right] d t^{\prime}\right\} \\
& =\frac{\epsilon}{\tau}\left[\frac{2 \pi}{\alpha}\right]^{1 / 2} e^{y_{0}^{2}}\left\{[ 1 - \frac { e ^ { - y _ { 0 } ^ { 2 } } } { \pi ^ { 1 / 2 } | y _ { 0 } | } + \frac { e ^ { - y _ { 0 } ^ { 2 } } } { 2 \pi ^ { 1 / 2 } | y _ { 0 } | ^ { 3 } } + \cdots ) \int _ { 0 } ^ { t } \operatorname { e x p } \left[-\left[\frac{\alpha}{2} t^{\prime 2}+\left(a_{0}+\tau^{-1}\right) t^{\prime}\right] d t^{\prime}\right.\right. \\
& -\int_{0}^{t_{s}} \Phi\left(\left|y\left(t^{\prime}\right)\right|\right) \exp \left[-\left[\frac{\alpha}{2} t^{\prime 2}+\left(a_{0}+\tau^{-1}\right) t^{\prime}\right]\right] d t^{\prime} \\
& \left.+\int_{t_{s}}^{t} \Phi\left(y\left(t^{\prime}\right)\right) \exp \left[-\left[\frac{\alpha}{2} t^{\prime 2}+\left(a_{0}+\tau^{-1}\right) t^{\prime}\right]\right] d t^{\prime}\right\}
\end{aligned}
$$

Since $t_{s} \gg \alpha^{-1 / 2}$, the rhs of (B4) results give, after some algebraic manipulation,

$\epsilon\left(\frac{\pi}{\alpha}\right)^{1 / 2} e^{a_{0}^{2} / \alpha}\left[1+\Phi\left(\left|a_{0}\right| \alpha^{-1 / 2}\right)\right]-\epsilon \tau+\epsilon \tau^{2}\left[a_{0}-\frac{(\pi \alpha)^{1 / 2}}{2} e^{a_{0}^{2} / \alpha}\left[1+\Phi\left(\left|a_{0}\right| \alpha^{-1 / 2}\right)\right]\right]+O\left(\left(\alpha^{1 / 2} \tau\right)^{3}\right)$.

Adding up the results (B2), (B3), and (B5), we obtain (4.5).

$$
\text { 2. } \alpha^{1 / 2} \tau \gg 1
$$

The first term $\left\langle h^{2}(0)\right\rangle$ is expanded in powers of $\left(\alpha^{1 / 2} \tau\right)^{-1}$ giving

$$
\frac{\epsilon}{a_{0}^{2} \tau}+\mathcal{O}\left(\left(\alpha^{1 / 2} \tau\right)^{-2}\right)
$$

The remaining contributions are treated using the same steps as for the integral term in (A6) and the expansion of $\Phi\left(z_{0}+z\right)$ around $z_{0}$ (see Appendix A)

$$
\begin{aligned}
& \epsilon\left[\Phi\left(x_{1}\right)-\Phi\left(x_{0}\right)\right] e^{x_{0}^{2}}\left[\Phi\left(\left|y_{0}\right|\right) \frac{\pi}{\alpha \tau} e^{y_{0}^{2}}+\frac{1}{1+\left|a_{0}\right| \tau}\left[\frac{2 \pi}{\alpha}\right]^{1 / 2}\right] \\
& +\frac{\epsilon}{\tau}\left(\frac{2 \pi}{\alpha}\right]^{1 / 2} e^{y_{0}^{2}} \int_{0}^{t} \Phi\left(y\left(t^{\prime}\right)\right) \exp \left[-\left[\frac{\alpha}{2} t^{\prime 2}+\left(a_{0}+\tau^{-1}\right) t^{\prime}\right]\right] d t^{\prime} \\
& =\frac{\epsilon}{\tau}\left[\Phi\left(a(t)(2 \alpha)^{-1 / 2}\right)+\Phi\left(\left|a_{0}\right|(2 \alpha)^{-1 / 2}\right)\right]\left[\frac{\pi}{\alpha} e^{a_{0}^{2} / \alpha} \Phi\left(\left|a_{0}\right|(2 \alpha)^{-1 / 2}\right)+\frac{1}{\left|a_{0}\right|}\left(\frac{2 \pi}{\alpha}\right)^{1 / 2} e^{a_{0}^{2} / 2 \alpha}\right] \\
& +\frac{\epsilon \pi}{\alpha \tau} e^{\left[\left(a_{0} \tau\right)^{2}-1\right] /\left(\alpha \tau^{2}\right)} \int_{y_{0}}^{y} e^{-2 \sqrt{2} y^{\prime} / \alpha^{1 / 2}} \Phi\left(y^{\prime}\right) \Phi^{\prime}\left(y^{\prime}\right) d y^{\prime} .
\end{aligned}
$$

Since $\left(\left|a_{0}\right| / \alpha\right)+(1 / \alpha \tau)<\tau / 2$, the rhs of (B7) can be conveniently manipulated to result in

$$
\begin{aligned}
\frac{\epsilon}{\tau}\left[\frac{\pi}{\alpha}\right]^{1 / 2}\{[ & {\left[\Phi\left(a(t)(2 \alpha)^{-1 / 2}\right)+\Phi\left(\left|a_{0}\right|(2 \alpha)^{-1 / 2}\right)\right]\left[\left[\frac{\pi}{\alpha}\right]^{1 / 2} e^{a_{0}^{2} / \alpha} \Phi\left(\left|a_{0}\right|(2 \alpha)^{-1 / 2}\right)+\frac{\sqrt{2}}{\left|a_{0}\right|} e^{a_{0}^{2} / 2 \alpha}\right] } \\
& \left.+\frac{1}{2}\left(\frac{\pi}{\alpha}\right]^{1 / 2} e^{a_{0}^{2} / \alpha}\left[1-\Phi^{2}\left(\left|a_{0}\right|(2 \alpha)^{-1 / 2}\right)\right]\right\}+\mathcal{O}\left(\left(\alpha^{1 / 2} \tau\right)^{-2}\right) .
\end{aligned}
$$

Adding up (B6) and (B8) for $t-\bar{t}>\alpha^{-1 / 2}$, we finally arrive at (4.8) with $B$ given by $B=\frac{\alpha^{1 / 2}}{a_{0}^{2}}+\left[\frac{2}{\left|a_{0}\right|}+\left[\frac{\pi}{2 \alpha}\right]^{1 / 2} e^{a_{0}^{2} / 2 \alpha}\left[1+\Phi\left(\left|a_{0}\right|(2 \alpha)^{-1 / 2}\right)\right]\right]\left[\frac{\pi}{2}\right]^{1 / 2} e^{a_{0}^{2} / 2 \alpha}\left[1+\Phi\left(\left|a_{0}\right|(2 \alpha)^{-1 / 2}\right)\right]+\mathcal{O}\left(\left(\alpha^{1 / 2} \tau\right)^{-1}\right)$ 
${ }^{1}$ P. Mandel, in Far from Equilibrium Phase Transitions, Vol. 319 of Lecture Notes in Physics, edited by L. Garrido (Springer, Berlin, 1988); P. Mandel, in Instabilities and Chaos in Quantum Optics II, edited by N. Abraham, F. T. Arecchi, and L. A. Lugiato (Plenum, New York, 1988). And references therein.

${ }^{2}$ W. Scharpf, M. Squicciarini, D. Bromley, C. Green, J. R. Tredicce, and L. M. Narducci, Opt. Commun. 63, 344 (1987).

${ }^{3}$ M. C. Torrent and M. San Miguel, Phys. Rev. A 38, 245 (1988).

${ }^{4}$ P. Spano, A. D'Ottavi, A. Mecozzi, and B. Daino, Appl. Phys. Lett. 52, 2203 (1988); A. Mecozzi, S. Piazzolla, A. D’Ottavi, and P. Spano, Phys. Rev. A 38, 3136 (1988).

${ }^{5}$ F. T. Arecchi, R. Meucci, and J. A. Roversi, Europhys. Lett. 8, 225 (1989); F. T. Arecchi, W. Gadomski, R. Meucci, and J. A. Roversi, Phys. Rev. A 39, 4004 (1989).

${ }^{6}$ A. Mecozzi, P. Spano, A. D'Ottavi, and S. Piazzolla (unpublished).

${ }^{7}$ B. Zambon (unpublished).

${ }^{8}$ C. Van den Broeck and P. Mandel, Phys. Lett. 122A, 36 (1987).

${ }^{9}$ H. Zeghlache, P. Mandel, and C. Van den Broeck, Phys. Rev.
A 40, 286 (1989).

${ }^{10}$ F. T. Arecchi and A. Politi, Phys. Rev. Lett. 45, 1219 (1980); F. T. Arecchi, A. Politi, and L. Ulivi, Nuovo Cimento 71, B 119 (1982).

${ }^{11}$ N. G. Stocks, R. Mannella, and P. V. E. McClintock (unpublished).

12J. M. Sancho and M. San Miguel, Phys. Rev. A 39, 2722 (1989).

${ }^{13}$ M. San Miguel, in Far from Equilibrium Phase Transitions, Vol. 319 of Lecture Notes in Physics, edited by L. Garrido (Springer, Berlin, 1988).

${ }^{14}$ H. J. Carmichael, Quantum Statistical Methods in Quantum Noise (Springer, Berlin, to be published).

${ }^{15}$ N. B. Abraham and D. E. Chyba, Proc. of Rochester Conference, edited by L. Mandel (1989). This paper contains a discussion of laser emission spectra below threshold associated with field or polarization noise.

${ }^{16}$ Handbook of Mathematical Functions, edited by $\mathbf{M}$. Abramowitz and I. Stegun (Dover, New York, 1970). 